EPJ Web of Conferences 60, 14007 (2013)

DOI: $10.1051 /$ epjconf $/ 20136014007$

(C) Owned by the authors, published by EDP Sciences, 2013

\title{
Jet and photon measurements from ATLAS
}

\author{
J. G. Saraiva ${ }^{a}$ On behalf of the ATLAS Collaboration
}

LIP (Laboratório de Instrumentação e Física Experimental de Particulas), Av. Elias Garcia 14-1², 1000-149 Lisboa, Portugal

\begin{abstract}
Differential cross-section measurements of inclusive-jet and di-jet production provide stringent tests of perturbative QCD predictions and provide inputs for determination of parton density functions. Ratios of jet multiplicities are sensitive to $\alpha_{s}$ and have reduced theoretical uncertainties. Measurements of the inclusive isolated-photon and di-photon cross-sections provide a direct probe of short-distance physics, complementary to that from measurements of jets or vector-bosons and are sensitive to the gluon density in the proton. The measurements are compared to next-to-leading-order or higher-order QCD calculations.
\end{abstract}

\section{Introduction}

ATLAS is a general purpose detector operating within the LHC experiment. The main goals of ATLAS are searches of the Higgs boson, study of the properties of the top quark and search for new physics. High-precision measurements to make novel tests of perturbative QCD (pQCD) will also be possible due to the higher centre-of-mass energy $(\sqrt{s})$ available at LHC than in previous accelerators. In this paper, measurements of jets and photons at ATLAS are presented. These measurements are one of the tools that can be used at LHC to increase the understanding of QCD interactions. In particular, they allow the testing of pQCD down to the shortest accessible distances. Their sensitivity to the proton parton distribution functions (PDFs) make them adequate to measure the strong coupling constant $\alpha_{s}$ and to the improvement of the determination of the PDFs. Photons are colorless probes to QCD and can be used to constrain the proton PDFs. Di-photons processes are in addition an important background for the identification of the Higgs boson in the $H \rightarrow \gamma \gamma$ channel. Comparison of data and next-to-leading-order (NLO) and next-to-next-leadingorder (NNLO) QCD theoretical calculations are the main focus of this paper.

\section{2 inclusive-jet measurements}

\section{1 cross-section for $\sqrt{s}=2.76 \mathrm{TeV}$}

The inclusive-jet cross-section was measured [1] using an integrated luminosity of $\mathcal{L}=0.20 \mathrm{pb}^{-1}$. Jets are reconstructed using the anti- $k_{t}$ algorithm with a distance parameter $R=0.6$. The jets were required to have a transverse momentum $p_{T} \subset[20,430] \mathrm{GeV}$ and rapidity $|y|<4$.4. The data are compared with theoretical calculations from NLO QCD predictions using NLOJET++ with the CT10 PDF set, to which non-perturbative corrections have been applied. Figure 1 shows the results for the double-differential jet cross-section $d \sigma^{2} / d p_{T} d y$. Figure 2 shows the ratios of the

a e-mail: gentil@lip.pt. Acknowledgments are due to GRICES and FCT. With the FCT grant SFRH/BPD/73474/2010.

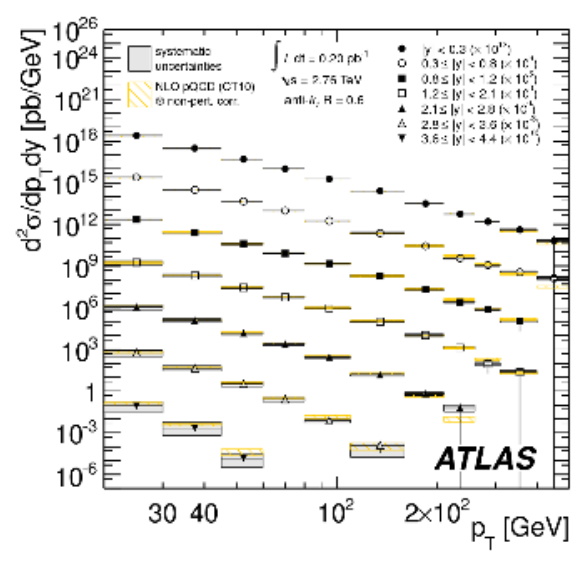

Fig. 1. Measured double-differential inclusive-jet cross sections as functions of $p_{T}$ in different regions of rapidity (markers). The measurements are scaled by the factors shown in parentheses to aid visibility. The shaded areas are the experimental systematic uncertainties. The hashed areas represent the QCD predictions and their uncertainties [1].

measured cross-section and the QCD predictions in different rapidity regions. Theoretical predictions using different PDF sets, namely MSTW 2008, NNPDF 2.1 and HERAPDF 1.5 and ABM $11 \mathrm{NLO}$, are also included in this figure. The agreement between data and theory is within $10 \%$ for $|y|<2.8$. For the two highest rapidity regions, $2.8<|y|<3.6$ and $3.6<|y|<4.4$, the agreement between data and theory is worse. The prediction based on the ABM 11 NLO PDF set gives a worse agreement for high $p_{T}$ values and the lowest rapidity regions. The measurements with $\sqrt{s}=2.76 \mathrm{TeV}$ are compared to those with $\sqrt{s}=7 \mathrm{TeV}$ [2] in the double ratio presented in Figure 3 . The small experimental uncertainty is due to the cancellation of the uncertainties between the two measurements. Figure 3 also includes other theoretical predictions with the different PDF sets already mentioned above. The data are generally slightly higher than the predictions in the central rapidity regions and slightly lower in the forward rapidity regions. A larger deviation is seen for ABM 11 NLO for the central rapidity region, as mentioned above. 

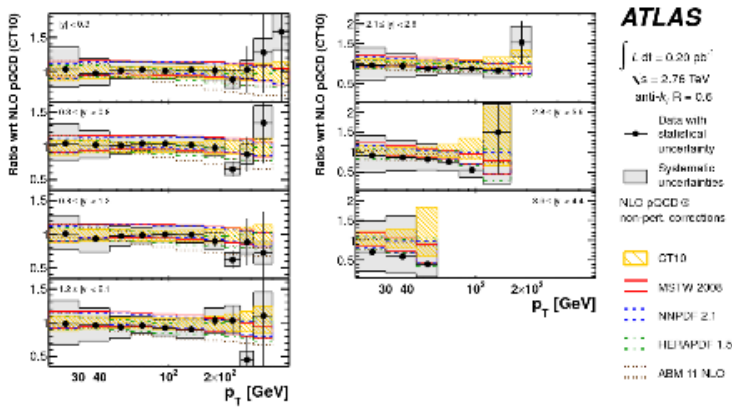

Fig. 2. Ratio of the measurements of Figure 1 and the predictions of NLOJET++ based on the CT10 PDF set. The predictions using different PDF sets are also shown. Other details as in the caption to Figure $1[1]$.
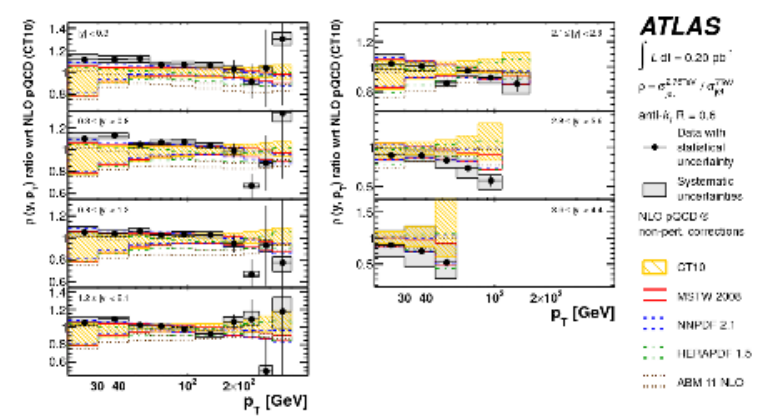

Fig. 3. Ratio of the inclusive-jet cross section at $\sqrt{s}=2.76 \mathrm{TeV}$ to the one at $\sqrt{s}=7 \mathrm{TeV}$, shown as double ratio to the theoretical prediction calculated with the CT10 PDFs as a function of $p_{T}$ in different rapidity regions. Theoretical predictions using different PDFs sets are also shown [1] .

\subsection{NLO QCD analysis combining HERA+ATLAS data}

The previous results have shown that due to the correlations at different center-of-mass energies, it is possible to increase the precision of the data. These can then be used to constrain the PDF uncertainties in a global fit within the NLO QCD framework. The analysis [1] was performed using a combination of data from the HERA experiments together with ATLAS data using the HERAFitter package with the DGLAP formalism and based on a $\chi^{2}$ minimization. In this analysis, care was taken to avoid regions of low $p_{T}$ and low $Q^{2}$, where the non-perturbative descriptions have a large uncertainty. The results of the fit for $x g(x)$ and $x S(x)$ are shown in Figures 4 and 5, respectively, at the scale $Q^{2}=1.9 \mathrm{GeV}^{2}$. The combination of ATLAS and HERA data results in a harder gluon distribution with smaller uncertainty at high Bjorken- $x$ than for HERA data alone. The sea quark distribution is softer at high Bjorken$x$ and has larger uncertainty.

\section{Di-jet cross-sections}

The di-jet cross-section was measured using data from 2011 with $\sqrt{s}=7 \mathrm{TeV}$, corresponding to an integrated luminosity of $\mathcal{L}=4.8 \mathrm{fb}^{-1}$ [3]. The jets were reconstructed using the anti- $k_{t}$ algorithm with distance parameter $R=0.6$. The leading jet was selected with $p_{T}>100 \mathrm{GeV}$ and

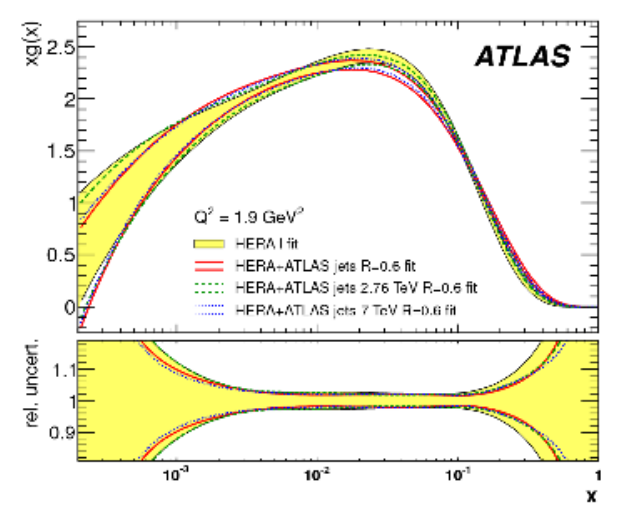

Fig. 4. The extracted gluon $x g(x)$ momentum distribution and the relative experimental uncertainty as a function of $x$ for $Q^{2}=1.9$ $\mathrm{GeV}^{2}[1]$

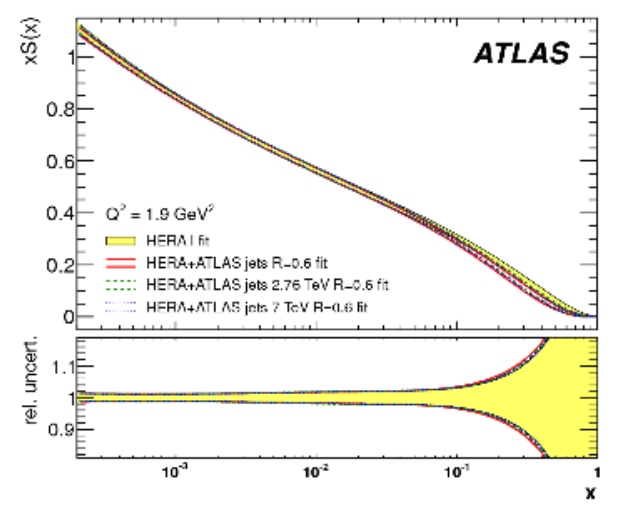

Fig. 5. The extracted sea quark $x S(x)$ momentum distribution and the relative experimental uncertainty as a function of $x$ for $Q^{2}=$ $1.9 \mathrm{GeV}^{2}[1]$.

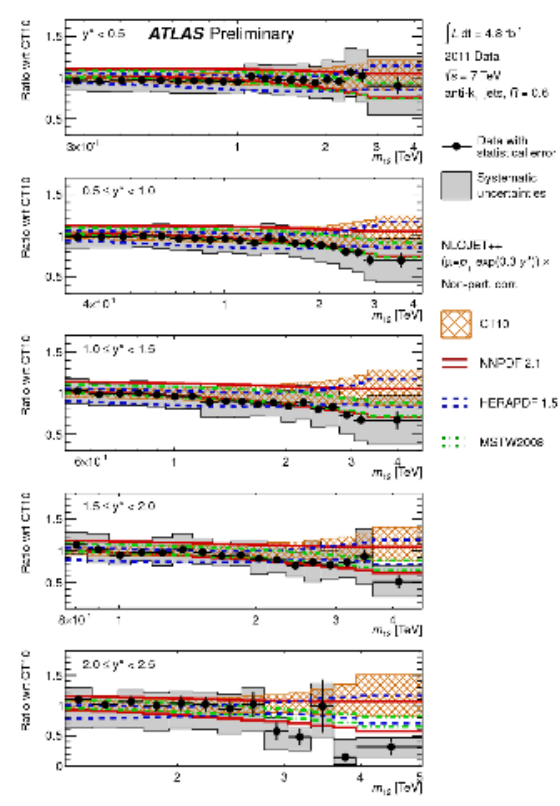

Fig. 6. Ratio of the measured double-differential di-jet cross section and NLOJET++ based on the CT10 PDF set as a function of the di-jet mass in different regions of $y^{*}$. The predictions based on different PDF sets are also included [3]. 


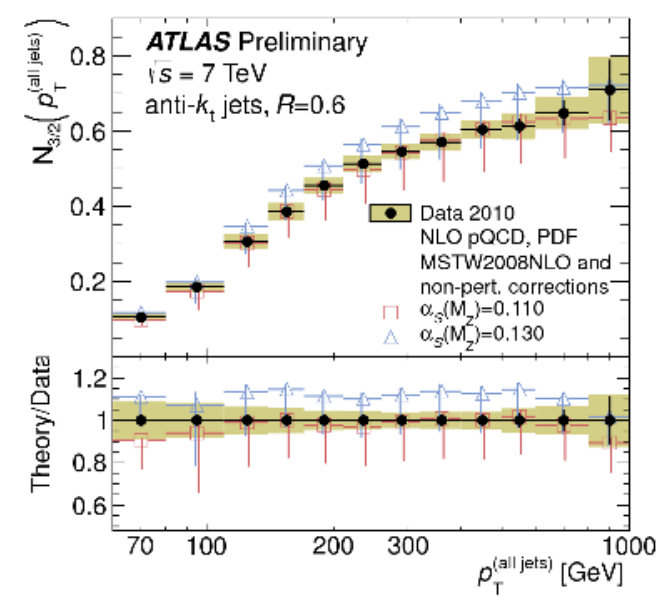

Fig. 7. The measured cross-section fraction $N_{3 / 2}$ as a function of $p_{T}$. The NLOJET++ predictions for $\alpha_{s}\left(M_{Z}\right)=0.110$ and 0.130 based on MSTW2008NLO and including non-perturbative corrections arealso included. The error bars in the predictions include the full theoretical uncertainty and are shifted to aid visibility [4].

the sub-leading jet was required to have $p_{T}>50 \mathrm{GeV}$. Both jets were required to have rapidities $|y|<2.8$. The dijet cross-section was measured as a function of the invariant mass and $y^{*}=\left|y_{j 1}-y_{j 2}\right| / 2$. The measurements span invariant masses from $260 \mathrm{GeV}$ to $4.6 \mathrm{TeV}$. Theoretical predictions calculated with NLOJET++ based on the CT10 PDF set and including non-perturbative corrections are compared to the data. Figure 6 shows the ratio between the measurements and the predictions. Comparisons with theoretical predictions using different PDF sets, namely MSTW2008, NNPDF 2.1 and HERAPDF 1.5, are also included in the figure. For all $y^{*}$ regions, data and theory, independently of the PDF set used, show a good agreement for $m_{12}<2.5 \mathrm{TeV}$. Above this value the theory predicts a higher cross-section.

\section{Multi-jet cross-sections and determination of $\alpha_{s}$}

The multi-jet cross-section was also measured using 2010 data with a $\sqrt{s}=7 \mathrm{TeV}$ [4]. Jets were reconstructed using the anti- $k_{t}$ algorithm with ae distance parameter $R=0.6$ and were required to have rapidities $|y|<2.8$. The leading jet was required to have $p_{T}>60 \mathrm{GeV}$ and the other jets should have $p_{T}>40 \mathrm{GeV}$. The ratio of cross-sections for multi-jets is a quantity sensitive to the strong coupling constant $\alpha_{s}$. The ratio

$$
N_{3 / 2}\left(p_{T}\right)=\frac{\sum_{i}^{n_{j e t}^{\prime}} d \sigma_{N_{j e t} \geq 3} / d p_{T, i}}{\sum_{i}^{n_{j e t}^{\prime \prime}} d \sigma_{N_{j e t} \geq 2} / d p_{T, i}}
$$

was measured as a function of $p_{T}$. The measured $N_{3 / 2}$ is presented in Figure 7. The NLO QCD predictions using NLOJET++ based on MSTW2008NLO including non-perturbative corrections with $\alpha_{s}\left(M_{Z}\right)=0.110$ and 0.130 are also shown in this figure. A least-squares fit to the measurements results in the value

$$
\alpha_{s}\left(M_{Z}\right)=0.111 \pm 0.006 \text { (exp.) }{ }_{-0.003}^{+0.016} \text { (theory). }
$$

Table 1. The values of $\alpha_{s}$ determined using different PDF sets in the NLO predictions. The uncertainty includes only the experimental source [4].

\begin{tabular}{ll}
\hline PDF & $\alpha_{s}$ \\
\hline MSTW08 & $0.111 \pm 0.006$ \\
CT10 & $0.109 \pm 0.006$ \\
HERAPDF 1.5 & $0.114 \pm 0.005$ \\
ABM 1.5 & $0.116 \pm 0.005$ \\
NNPDF 2.3 & $0.112 \pm 0.005$ \\
\hline
\end{tabular}

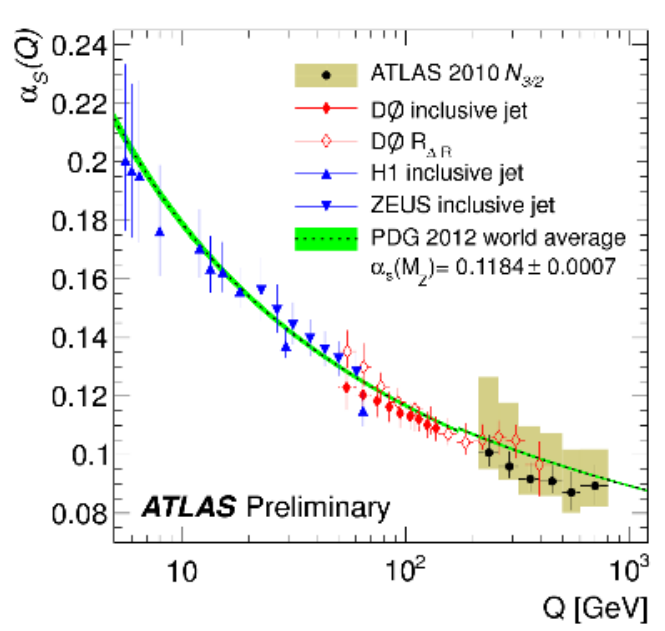

Fig. 8. The strong coupling constant $\alpha_{s}$ from ATLAS [4], DØ [8], ZEUS [9] and $\mathrm{H} 1$ [10] as a function of the scale $Q$. For ATLAS, the error bars correspond to the experimental uncertainties while the shaded region is the total uncertainty. For the other experiments the error bars correspond to the total uncertainty [4].

This result is in agreement with the world average and with determinations from other experiments:

$$
0.1184 \pm 0.0007 \text { (world average) [5] }
$$

$$
\begin{gathered}
0.1178 \pm 0.0001 \text { (stat.) }{ }_{-0.0095}^{+0.0081} \text { (syst.) (CDF) [7] } \\
0.1191_{-0.0071}^{+0.0048}(\mathrm{D} \varnothing)[8]
\end{gathered}
$$

In Table 1 the results of $\alpha_{s}$ using different PDF sets show an independence on the PDF input used in the theoretical predictions. The results for the running of $\alpha_{s}$ are shown in Figure 8. ATLAS data covers the region of Q $\subset[200,800]$ $\mathrm{GeV}$, compatible with the predictions from the Renormalization Group Equation (RGE) [6] based on the world average $\alpha_{s}$.

\section{Isolated-photon cross-sections}

Measurements of the isolated-photon differential cross-sections as functions of $E_{T}^{\gamma}$ and $\eta^{\gamma}$ were made using 2011 data with an intergrated luminosity of $\mathcal{L}=4.7 \mathrm{fb}^{-1}$ for $\sqrt{s}=7 \mathrm{TeV}$ [11]. The photons were selected with $100<$ $E_{T}^{\gamma}<1000 \mathrm{GeV}$ and $\left|\eta^{\gamma}\right|<1.37$ and $1.52 \leq\left|\eta^{\gamma}\right| \leq 2.37$. The data are compared to NLO and LO theoretical predictions: (i) NLO QCD JeTPHOx 1.3 + BFG set II that includes direct and fragmentation processes and using two proton PDF sets, namely CT10 and MSTW2008 NLO (ii) leading-logarithm parton shower predictions of Рутніа 6.4 


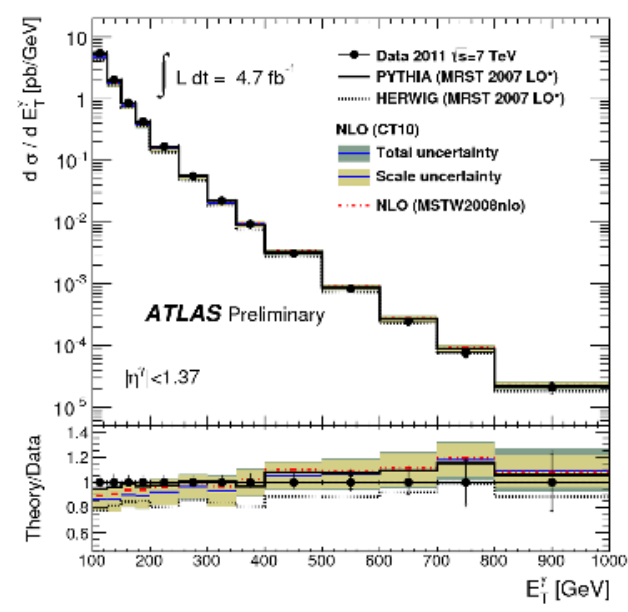

Fig. 9. Measured isolated-photon differential cross section as a function of $E_{T}^{\gamma}$. The inner error bars show statistical uncertainties while the outer bars are the full uncertainties. The LO and NLO theoretical predictions detailed in the text are also included. The shaded region represent the full theoretical uncertainty [11].

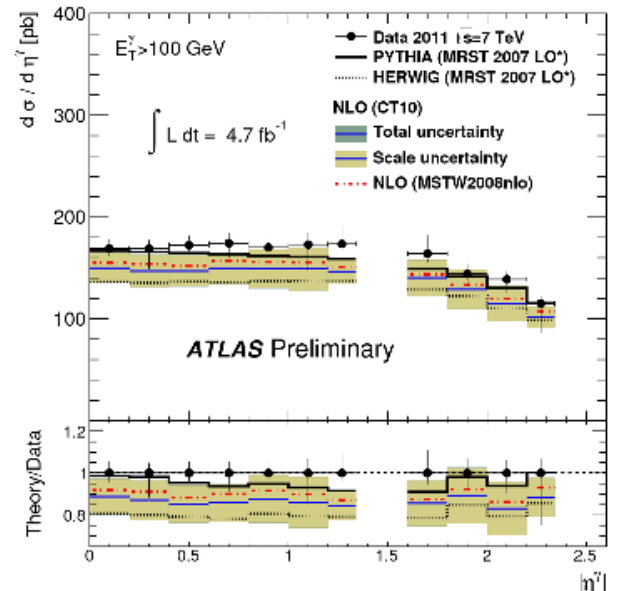

Fig. 10. Measured isolated-photon differential cross section as a function of $\eta^{\gamma}$. Other details as in the caption to Figure 9 [11].

and Herwig 6.5, which include the $2 \rightarrow 2$ processes $q g \rightarrow$ $q \gamma$ and $q \bar{q} \rightarrow q \gamma$, based on MRST2007LO proton PDF sets. The AMBT2 and AUET2 tunes were used for PythIA and Herwig, respectively. Figure 9 shows the differential cross-section as a function of $E_{T}^{\gamma}$. The theoretical predictions describe the data. Figure 10 shows the differential cross-section as a function of $\eta^{\gamma}$; the theoretical predictions underestimate the measurements in all the regions considered. The measured integrated cross-section for isolatedphotons is $\sigma(p p \rightarrow \gamma+X)=234 \pm 2$ (stat) $)_{-9}^{+13}$ (syst) \pm 4 (lumi) pb for $\left|\eta^{\gamma}\right|<1.37$ and $\sigma(p p \rightarrow \gamma+X)=122 \pm$ 2 (stat) - $_{-7}$ (syst) \pm 2 (lumi) pb for $1.52<\left|\eta^{\gamma}\right|<2.37$. Table 1 summarizes the total cross-section for these two pseudorapidity regions for the four theoretical predictions considered in this study.

\section{Di-photon cross-sections}

For the di-photon cross-section measurements [12], the complete 2011 data set of $\sqrt{s}=7 \mathrm{TeV}$ was used, corresponding to $\mathcal{L}=4.9 \mathrm{fb}^{-1}$. The photon selection required two
Table 2. Predicted integrated cross sections for isolated-photon production [11].

\begin{tabular}{lcc}
\hline Model & \multicolumn{2}{c}{$\sigma(p p \rightarrow \gamma+X)(\mathrm{pb})$} \\
& $|\eta|<1.37$ & $1.52<|\eta|<2.37$ \\
\hline PYTHIA & 224 & 118 \\
HERWIG & 187 & 99 \\
JETPHOX CT10 & $203 \pm 25$ & $105 \pm 15$ \\
JETPHOX MSTW2008 & $212 \pm 24$ & $109 \pm 15$ \\
\hline
\end{tabular}

Table 3. Predicted integrated cross sections for isolated-photon pair production [12].

\begin{tabular}{lc}
\hline Model & $\sigma(p p \rightarrow \gamma \gamma+X)(\mathrm{pb})$ \\
\hline 2 $\gamma$ NNLO MSTW2008 NNLO & $44_{-5}^{+6}$ \\
DIPHOX+GAMMA2MC CT10 NLO & $39_{-6}^{+7}$ \\
PYTHIA MRST2007LO & 36 \\
SHERPA CTEQ6L1 & 36 \\
\hline
\end{tabular}

isolated-photons with $E_{T}^{\gamma}>25(22) \mathrm{GeV}$ for the (sub-)leading photon and an angular separation of $\Delta R>0.4$ and within the same pseudorapidity range mentioned in Section 5 . The measured integrated cross-section measured is $\sigma(p p \rightarrow$ $\gamma \gamma+X)=44_{-4.2}^{+3.2} \mathrm{pb}$. The theoretical predictions are shown in Table 3. The LO models underestimate the data by $20 \%$. The NLO Diphox+Gamma2MC prediction underestimates the data by $10 \%$ whereas the $2 \gamma$ NNLO reproduces the data well. Figures 11 and 12 show the measured differential
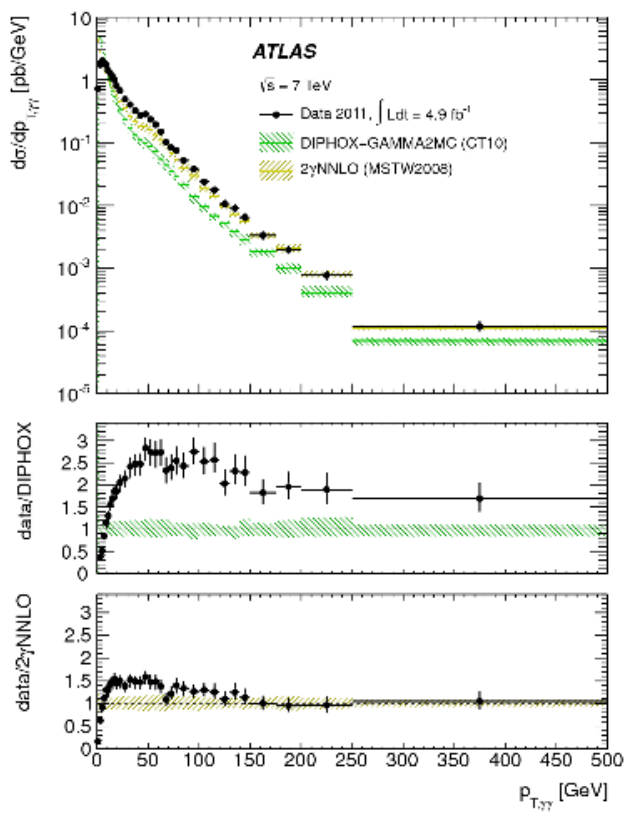

Fig. 11. Measured differential cross section as a function of $p_{T}^{\gamma \gamma}$. The predictions from Diphox+Gamma2MC and $2 \gamma$ NNLO are also shown [12].

cross-sections as functions of $p_{T, \gamma \gamma}$ and $\Delta \phi_{\gamma \gamma}$ together with the higher-order theoretical predictions. The DiPHox calculation underestimates the data in the full range considered whereas $2 \gamma \mathrm{NNLO}$ prediction is much closer to the measured results, except in the regions where the fragmenta- 

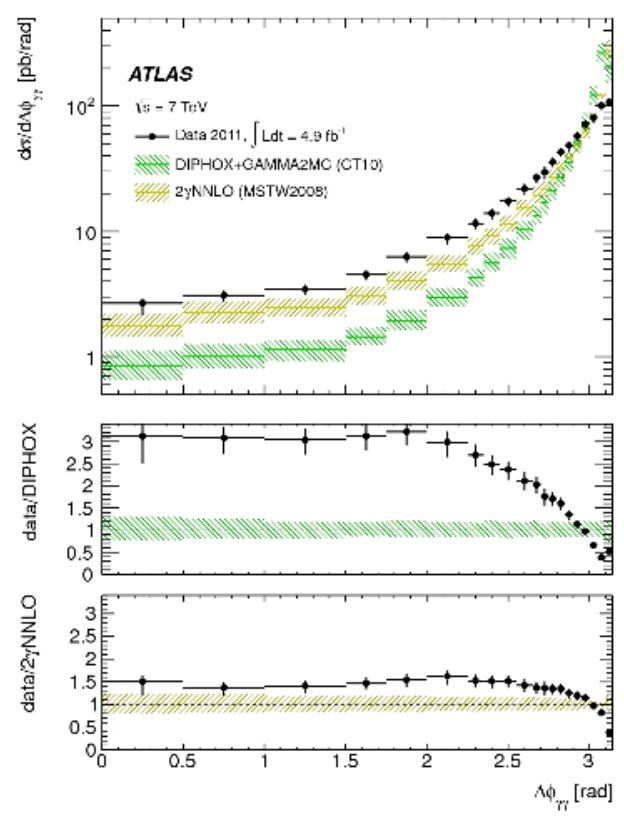

Fig. 12. Measured differential cross section as a function of $\Delta \phi_{\gamma \gamma}$. Other details as in the caption to Figure 11 [12]

tion component (not included in this calculation) is still significant.

\section{References}

1. ATLAS Collaboration, CERN-PH-EP-2013-036 (submitted to: Eur. Phys. J. C) [arXiv:1304.4739v1], 2013

2. ATLAS Collaboration, Phys. Rev. D 86 (2012) 014022 , arXiv:1112.6297

3. ATLAS Collaboration, ATLAS-CONF-2012-021, 2012 (http://cds.cern.ch/record/1430730/files/ ATLAS-CONF-2012-021.pdf)

4. ATLAS Collaboration, ATLAS-CONF-2013-041, 2013 (http: //cds . cern.ch/record/1543225/files/ ATLAS-CONF-2013-041.pdf)

5. S. Bethke, Eur. Phys. J.C64(2009) 689-703

6. J. Beringer et al., Review of Particle Physics, Phys.Rev.D86(2012) 010001

7. T. Affolder et al., Phys. Lett. 88(2002) 042001

8. V. M. Abazov et al., Phys. Rev. D 80 (2009) 111107

9. H. Abramowicz et al., Nucl.Phys. B864 (2012) 1

10. Aktas, A. et al., Eur.Phys.J. C65 (2010) 363-383

11. ATLAS Collaboration, ATLAS-CONF-2013-022, 2013 (http://cds.cern.ch/record/1525723/ files/ATLAS-CONF-2013-022.pdf)

12. ATLAS Collaboration, CERN-PH-EP-2012-300 (submitted to: JHEP) [arXiv:1211.1913v2], 2013 